STRUCTURAL BIOLOGY COMMUNICATIONS

ISSN 2053-230X

Received 1 November 2021

Accepted 8 December 2021

Edited by J. Newman, Bio21 Collaborative

Crystallisation Centre, Australia

Keywords: oxidoreductases; short-chain dehydrogenase/reductase family; dihydrofolate reductases; NADPH; Brucella suis; Brucella canis; Seattle Structural Genomics Center for Infectious Disease; SSGCID.

PDB references: BsFolM, 5tgd; BcFolM, 5bt9

Supporting information: this article has supporting information at journals.iucr.org/f

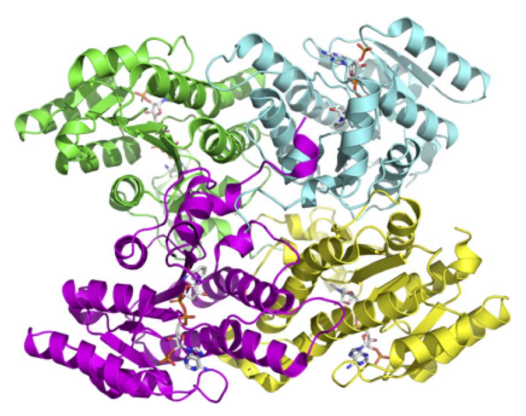

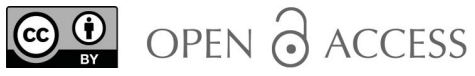

\section{Crystal structures of FolM alternative dihydrofolate reductase 1 from Brucella suis and Brucella canis}

Imani Porter, ${ }^{a}$ Trinity Neal, ${ }^{a}$ Zion Walker, ${ }^{a}$ Dylan Hayes, ${ }^{\text {a }}$ Kayla Fowler, ${ }^{a}$ Nyah Billups, ${ }^{a}$ Anais Rhoades, ${ }^{a}$ Christian Smith, ${ }^{a}$ Kaelyn Smith, ${ }^{a}$ Bart L. Staker, b,c David M. Dranow, ${ }^{\text {b,d }}$ Stephen J. Mayclin, ${ }^{b, d}$ Sandhya Subramanian, ${ }^{b, c}$ Thomas E. Edwards, ${ }^{\text {b,d }}$ Peter J. Myler ${ }^{\mathrm{b}, \mathrm{c}}$ and Oluwatoyin A. Asojo ${ }^{\mathrm{a} *}$

${ }^{\mathrm{a}}$ Department of Chemistry and Biochemistry, Hampton University, 100 William R. Harvey Way, Hampton, VA 23668, USA, ${ }^{\mathbf{b}}$ Seattle Structural Genomics Center for Infectious Disease (SSGCID), Seattle, Washington, USA, ${ }^{\mathbf{c}}$ Center for Infectious Disease Research, formerly Seattle Biomedical Research Institute, 307 Westlake Avenue North Suite 500, Seattle, Washington, USA, and ${ }^{\mathbf{d} B e r y l l i u m ~ D i s c o v e r y, ~ B a i n b r i d g e ~ I s l a n d, ~ W A ~ 98110, ~ U S A . ~ * C o r r e s p o n d e n c e ~ e-m a i l: ~}$ oluwatoyin.asojo@hamptonu.edu

Members of the bacterial genus Brucella cause brucellosis, a zoonotic disease that affects both livestock and wildlife. Brucella are category B infectious agents that can be aerosolized for biological warfare. As part of the structural genomics studies at the Seattle Structural Genomics Center for Infectious Disease (SSGCID), FolM alternative dihydrofolate reductases 1 from Brucella suis and Brucella canis were produced and their structures are reported. The enzymes share $\sim 95 \%$ sequence identity but have less than $33 \%$ sequence identity to other homologues with known structure. The structures are prototypical NADPH-dependent short-chain reductases that share their highest tertiarystructural similarity with protozoan pteridine reductases, which are being investigated for rational therapeutic development.

\section{Introduction}

Brucellosis is the most common bacterial zoonotic disease and is caused by the bacterial genus Brucella, which infects humans who consume contaminated animal products, or through contact with infected animals and their secretions (Ducrotoy et al., 2016; Godfroid, Al Dahouk et al., 2013). Brucella are classified as category B infectious agents that can be aerosolized (de Figueiredo et al., 2015). Serological evidence suggests that human brucellosis is misdiagnosed as malaria or other febrile diseases in sub-Saharan Africa (Ducrotoy et al., 2017). Brucellosis is highly contagious and affects economically important livestock and wild animals globally (Ducrotoy et al., 2017; Godfroid, Garin-Bastuji et al., 2013; Godfroid et al., 2011; Megersa et al., 2011). While brucellosis has been eradicated in cattle and small ruminants in a few countries, it remains endemic globally within a wide range of animal hosts (Moreno, 2014).

Current control approaches for brucellosis include vaccination, education and basic hygiene; however, these strategies have not effectively reduced the disease burden due to cost and other issues (Ariza et al., 2007). Notably, current vaccines are species-specific and are devastating to pregnant livestock, and cultural practices among rural dwellers and nomadic groups that rear animals are often incompatible with disease control (Ducrotoy et al., 2017; Godfroid, Al Dahouk et al., 2013). There is a continued need to develop new cost-effective approaches to treat infected animals, including the rational 
Table 1

Production of FolM alternative dihydrofolate reductase 1 from $B$. suis.

\begin{tabular}{|c|c|}
\hline Source organism & Brucella suis 1330 \\
\hline DNA source & $\begin{array}{l}\text { Dr Jean-Jacques Letesson (University of } \\
\text { Namur, Belgium) }\end{array}$ \\
\hline Forward primer & $\begin{array}{l}5^{\prime}-\text { CTCACCACCACCACCACCATATGGTGT } \\
\text { TGAATGATCCCGAAGC- } 3^{\prime}\end{array}$ \\
\hline Reverse primer & $\begin{array}{l}\text { 5'-ATCCTATCTTACTCACTTATTCGGTAA } \\
\text { TTCCTGCAATGTCGG-3' }\end{array}$ \\
\hline Expres & pBG1861 \\
\hline Expression host & E. coli BL21(DE3)R3 Rosetta cells \\
\hline $\begin{array}{l}\text { Complete amino-acid sequence } \\
\text { of the construct produced }\end{array}$ & $\begin{array}{l}\text { MAHHHHHHMLNDPEARMVANCPVLVTGGAR } \\
\text { RIGKAIVEDLASHGFPVIHCNRSLDEG } \\
\text { EAIANRINDSGGNACVVQADLEGDVRGL } \\
\text { VKQASDRIGPIRLLVNNASLFQEDKVGA } \\
\text { LDMALWDRHFAVHLKTPVILAEDMRKAL } \\
\text { PEDQDGLVVNI IDQRVWKLNPQFSYTL } \\
\text { SKSALWNATRTLAQALAPRIRVNAIAPG } \\
\text { PTLPSERQRPEDFERQVSKLPLQRAPEL } \\
\text { PEFGRTVRYFWENRSITGQMIALDGGQH } \\
\text { LAWETPDIAGITE }\end{array}$ \\
\hline
\end{tabular}

design or repurposing of small molecules that target enzymes that are vital for bacterial survival. The Seattle Structural Genomics Center for Infectious Disease (SSGCID) has determined the crystal structures of many target enzymes, including FolM alternative dihydrofolate reductase 1 from two Brucella species, B. suis and B. canis. Dihydrofolate reductase reduces dihydrofolic acid to tetrahydrofolic acid using reduced nicotinamide adenine dinucleotide phosphate (NADPH) as the electron donor. While this reaction is catalyzed by the enzyme dihydrofolate reductase (DHFR) in mammals and other organisms, some bacteria have an alternative pathway for reduced folate biosynthesis using FolM alternative dihydrofolate reductase 1 (Levin et al., 2004). Here, we present the crystal structures of FolM alternative dihydrofolate reductase 1 from two Brucella species, B. suis (BsFolM) and B. canis (BcFolM).

$B s$ FolM and $B c$ FolM are $95 \%$ identical in sequence. $B L A S T$ alignment of the protein sequences against the Protein Data Bank (PDB) reveals the most similar proteins to be Tt0495 from Thermus thermophilus HB8 (Pampa et al., 2014 ) with $\sim 32 \%$ sequence identity and $\sim 85 \%$ coverage; Leishmania major pteridine reductase (Schüttelkopf et al., 2005 ) with $\sim 30 \%$ sequence identity and $~ 90 \%$ coverage; Mycobacterium smegmatis short-chain reductase (Blaise et al., 2017 ) with $\sim 33 \%$ sequence identity and $\sim 85 \%$ coverage; and Trypanosoma cruzi pteridine reductase 2 (Schormann et al., 2005 ) with $\sim 30 \%$ sequence identity and $\sim 88 \%$ coverage. The reported crystal structures of $B s$ FolM and $B c$ FolM are the first steps towards identifying new therapeutics for brucellosis.

\section{Materials and methods}

\subsection{Macromolecule production}

Cloning, expression and purification were conducted as part of the Seattle Structural Genomics Center for Infectious Disease (SSGCID) following standard protocols described previously (Myler et al., 2009; Stacy et al., 2011; Bryan et al., 2011; Choi et al., 2011; Serbzhinskiy et al., 2015). The full-
Table 2

Production of FolM alternative dihydrofolate reductase 1 from B. canis.

\begin{tabular}{ll}
\hline Source organism & Brucella canis RM-666 (NCTC 10854) \\
DNA source & ATCC 23365 \\
Forward primer & 5'-CTCACCACCACCACCACCATATGGTGT \\
& TGAATGATCCCGAAGC-3' \\
Reverse primer & 5'-ATCCTATCTTACTCACTTATTCGGTAA \\
& TTCCTGCAATGTCGG-3' \\
Expression vector & pBG1861 \\
Expression host & E. coli BL21(DE3)R3 Rosetta cells \\
Complete amino-acid sequence & MAHHHHHMLNDPEARMVANCPVLVTGGA \\
\multicolumn{1}{c}{ of the construct produced } & RRIGKAIVEDLASHGFPVAIHCNRSLDE \\
& GEAIANRINDSGGNACVVQADLEGDVRG \\
& LVKQASDRIGPIRLLVNNASLFQEDKVG \\
& ALDMALWDRHAVHLKTPVILAEDMRKA \\
& LPEDQDGLVVNIDQRVWKLNPQFFSY \\
& LSKTALWNATRTLAQALAPRIRVNAIAP \\
& GPTLPSERQRPEDFERQVSKLPLQRAPE \\
& LPEFGRTVRYFENRS ITGQMIALDGGQ \\
& HLAWETPDIAELPNK \\
\hline
\end{tabular}

length FolM genes from B. suis (UniProt A0A0H3G2T6) and B. canis (UniProt A9MA73) were PCR-amplified from genomic DNA using the primers shown in Tables 1 and 2, respectively. The resultant amplicons were cloned into the ligation-independent cloning (LIC; Aslanidis \& de Jong, 1990) expression vector pBG1861 encoding a noncleavable $6 \times$ His fusion tag (MAHHHHHHM-ORF). The plasmids containing A0A0H3G2T6 and A9MA73 were tested for expression and 21 of culture was grown using auto-induction medium (Studier, 2005) in a LEX Bioreactor (Epiphyte Three). The expression clones for BrsuA.00010.a.B1.GE36748 and BrcaA.00010.a.B1.GE38297 are available at https:// www.ssgcid.org/available-materials/expression-clones/.

His- $B s$ FolM and His- $B c$ FolM were purified in a two-step protocol consisting of an $\mathrm{Ni}^{2+}$-affinity chromatography step and size-exclusion chromatography (SEC). All chromatography runs were performed on an ÄKTApurifier 10 (GE) using automated IMAC and SEC programs according to previously described procedures (Bryan et al., 2011). Thawed bacterial pellets were lysed by sonication in $200 \mathrm{ml}$ lysis buffer [25 m $M$ HEPES pH 7.0, $500 \mathrm{~m} M \mathrm{NaCl}, 5 \%$ glycerol, $0.5 \%$ CHAPS, $30 \mathrm{~m} M$ imidazole, $10 \mathrm{~m} M \mathrm{MgCl}_{2}, 1 \mathrm{~m} M$ tris(2carboxyethyl)phosphine (TCEP), $250 \mu \mathrm{g} \mathrm{ml}^{-1}$ 4-benzenesulfonyl fluoride hydrochloride (AEBSF), $0.025 \%$ azide]. After sonication, the crude lysate was clarified with $20 \mu \mathrm{l}$ ( 25 units $\mu^{-1}$ ) benzonase and incubated while mixing at room temperature for $45 \mathrm{~min}$. The lysate was then clarified by centrifugation at $10000 \mathrm{rev} \min ^{-1}$ for $1 \mathrm{~h}$ using a Sorvall centrifuge (Thermo Scientific). In the IMAC step, the clarified supernatant was passed over an Ni-NTA HisTrap FF $5 \mathrm{ml}$ column (GE Healthcare) pre-equilibrated with loading buffer (25 $\mathrm{m} M$ HEPES pH 7.0, $500 \mathrm{~m} M \mathrm{NaCl}, 5 \%$ glycerol, $30 \mathrm{~m} M$ imidazole, $1 \mathrm{~m} M$ TCEP, $0.025 \%$ sodium azide). The column was washed with 20 column volumes $(\mathrm{CV})$ of loading buffer and eluted with a linear gradient over $7 \mathrm{CV}$ of loading buffer plus $250 \mathrm{~m} M$ imidazole. Peak fractions, as determined by UV at $280 \mathrm{~nm}$, were pooled and concentrated. A SEC column (Superdex 75, GE Healthcare) was equilibrated with running buffer (25 m $M$ HEPES pH 7.0, $500 \mathrm{~m} M \mathrm{NaCl}, 5 \%$ glycerol, 
Table 3

Crystallization of FolM alternative dihydrofolate reductase 1 from $B$. suis (BsFolM).

\begin{tabular}{ll}
\hline Method & Vapor diffusion, sitting drop \\
Plate type & Rigaku Reagents XJR \\
Temperature (K) & 290 \\
Crystallization & BsFolM $\left(19 \mathrm{mg} \mathrm{ml}^{-1}\right)$ incubated with 4 mM \\
& NADPH, mixed 1:1 with MCSG1 condition A1 \\
& {$[20 \%(w / v)$ PEG 8000, 100 mM HEPES pH 7.5] } \\
Composition of reservoir & $20 \%(w / v)$ PEG 8000, 100 mM HEPES pH 7.5 \\
$\quad$ solution & \\
Volume and ratio of drop & $0.4 \mu \mathrm{l}: 0.4 \mu \mathrm{l}$ \\
Volume of reservoir $(\mu \mathrm{l})$ & 80
\end{tabular}

Table 4

Crystallization of FolM alternative dihydrofolate reductase 1 from B. canis (BcFolM).

\begin{tabular}{ll}
\hline Method & Vapor diffusion, sitting drop \\
Plate type & Rigaku Reagents XJR \\
Temperature $(\mathrm{K})$ & 290 \\
Crystallization & BcFolM $\left(32.3 \mathrm{mg} \mathrm{ml}^{-1}\right)$ incubated with $6 \mathrm{~m} M$ \\
& NADPH, mixed 1:1 with $20 \%(w / v)$ PEG 8000, \\
& $100 \mathrm{~m} M$ HEPES pH 7.5 \\
Composition of reservoir & $20 \%(w / v)$ PEG 8000, $100 \mathrm{~m} M$ HEPES pH 7.5 \\
$\quad$ solution & \\
Volume and ratio of drop & $0.4 \mu \mathrm{l}: 0.4 \mu \mathrm{l}$ \\
Volume of reservoir $(\mu \mathrm{l})$ & 80 \\
\hline
\end{tabular}

$2 \mathrm{~m} M$ DTT, $0.025 \%$ azide). The peak fractions were collected and analyzed by SDS-PAGE. The SEC peak fractions eluted as a single large peak at a molecular mass of $\sim 77 \mathrm{kDa}$, suggesting an oligomer, most likely dimeric, trimeric or tetrameric enzyme. The peak fractions were pooled and concentrated to $28.5 \mathrm{mg} \mathrm{ml}^{-1}$ (His-BsFolM) or $32.3 \mathrm{mg} \mathrm{ml}^{-1}$ (His- $B c$ FolM) as assessed by the $\mathrm{OD}_{280}$ using an Amicon concentration system (Millipore). Aliquots of $200 \mu \mathrm{l}$ were flash-frozen in liquid nitrogen and stored at $-80^{\circ} \mathrm{C}$ until use for crystallization.
Table 5

Data-collection and processing statistics for FolM alternative dihydrofolate reductase 1 from $B$. suis (PDB entry $5 \operatorname{tgd}, B s$ FolM) and $B$. canis (PDB entry 5bt9, BcFolM).

\begin{tabular}{|c|c|c|}
\hline PDB code & $5 \operatorname{tgd}$ & $5 b t 9$ \\
\hline Diffraction source & $\begin{array}{l}\text { APS beamline } \\
\text { 21-ID-F }\end{array}$ & $\begin{array}{l}\text { APS beamline } \\
21-\text { ID-F }\end{array}$ \\
\hline Wavelength $(\AA)$ & 0.97872 & 0.97872 \\
\hline Temperature (K) & 100 & 100 \\
\hline Detector & $\begin{array}{l}\text { RayoniX MX-300 } \\
\text { CCD }\end{array}$ & $\begin{array}{l}\text { MAR Mosaic } 225 \mathrm{~mm} \\
\text { CCD }\end{array}$ \\
\hline $\begin{array}{l}\text { Crystal-to-detector distance } \\
(\mathrm{mm})\end{array}$ & 220 & 130 \\
\hline Rotation range per image $\left(^{\circ}\right)$ & 1 & 1 \\
\hline Total rotation range $\left({ }^{\circ}\right)$ & 200 & 220 \\
\hline Space group & $P 2_{1}$ & $P 2_{1}$ \\
\hline$a, b, c(\AA)$ & $76.35,76.52,98.26$ & $76.57,75.60,99.18$ \\
\hline$\alpha, \beta, \gamma\left({ }^{\circ}\right)$ & $90,109.47,90$ & $90,109.23,90$ \\
\hline Mosaicity $\left({ }^{\circ}\right)$ & 0.180 & 0.168 \\
\hline Resolution range $(\AA)$ & $50-1.70(1.74-1.70)$ & $50.0-1.50(1.54-1.50)$ \\
\hline Total No. of reflections & $491527(36146)$ & $783894(57336)$ \\
\hline No. of unique reflections & $116233(8502)$ & 164992 (11908) \\
\hline Completeness (\%) & $99.0(98.4)$ & $96.4(94.6)$ \\
\hline Multiplicity & $4.22(4.25)$ & $4.8(4.8)$ \\
\hline$\langle I / \sigma(I)\rangle$ & $19.84(2.87)$ & $18.26(3.39)$ \\
\hline$R_{\text {r.i.m. }} \dagger$ & $0.050(0.486)$ & $0.053(0.553)$ \\
\hline $\begin{array}{l}\text { Overall } B \text { factor from Wilson } \\
\quad \text { plot }\left(\AA^{2}\right)\end{array}$ & 18.85 & 15.63 \\
\hline
\end{tabular}

$\dagger$ Estimated $R_{\text {r.i.m. }}=R_{\text {merge }}[N /(N-1)]^{1 / 2}$, where $N$ is the data multiplicity.

solution $(0.4 \mu \mathrm{l})$ and precipitant solution were set up at $290 \mathrm{~K}$ against $80 \mu \mathrm{l}$ reservoir in sitting-drop vapor-diffusion format. Before crystallization, NADPH was added to the protein solution to a final concentration of $4 \mathrm{~m} M(B s$ FolM) or $6 \mathrm{~m} M$ ( $B c$ FolM). The precipitant solution was MCSG-1 condition A1 (Tables 3 and 4). The crystals were harvested and cryoprotected with crystallization solution supplemented with $20 \%$ ethylene glycol before flash-cooling in liquid nitrogen.

\subsection{Crystallization}

Purified His-BsFolM and His-BcFolM were screened for crystallization in 96-well sitting-drop plates against the JCSG++ HTS (Jena Bioscience) and MCSG1 (Molecular Dimensions) crystallization screens. Equal volumes of protein

\subsection{Data collection and processing}

Data were collected at $100 \mathrm{~K}$ at the Advanced Photon Source, Argonne National Laboratory (Table 5). The data were reduced with XSCALE (Kabsch, 2010). Raw X-ray diffraction images are available at the Integrated Resource for

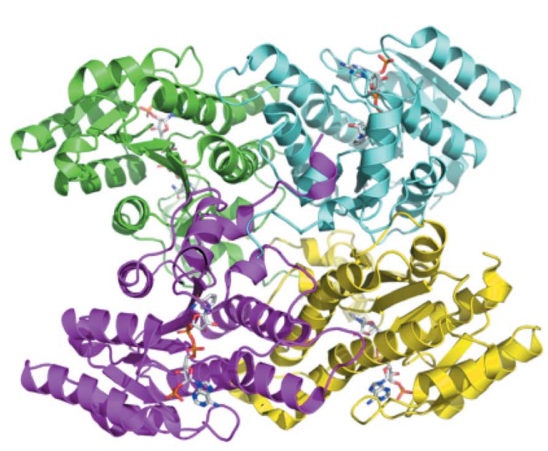

(a)

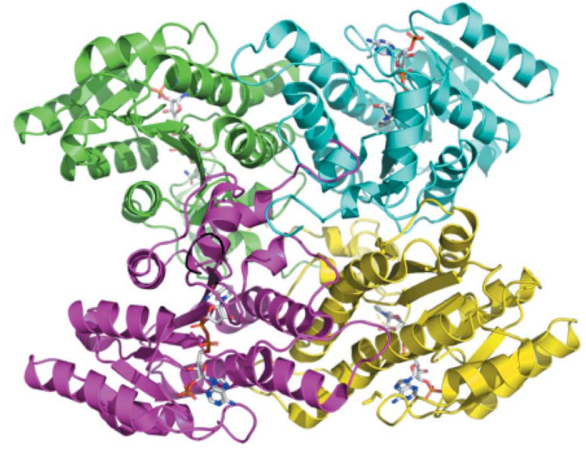

(b)

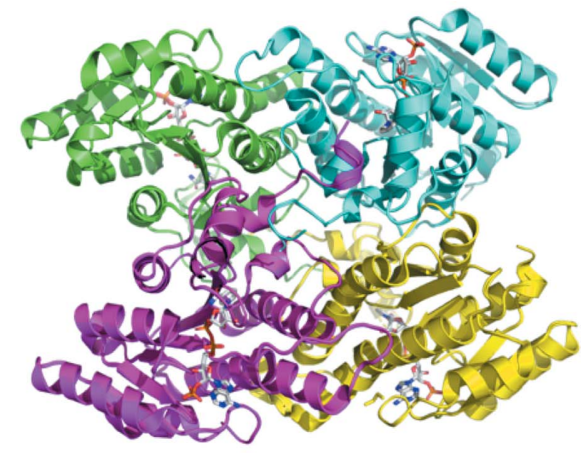

(c)

Figure 1

(a) $B s$ FolM and $(b) B c$ FolM assemble as prototypical FolM alternative dihydrofolate reductase 1 tetramers. $(c)$ The $B s$ FolM and $B c$ FolM tetramers are almost identical based on their structural alignment. 
Reproducibility in Macromolecular Crystallography at https:// www.proteindiffraction.org/.

\subsection{Structure solution and refinement}

Both structures were solved by molecular replacement. $B c$ FolM was solved with BALBES (Long et al., 2008) with PDB entry 2 uvd, a 3-oxoacyl-(acyl carrier protein) reductase
(Ba3989) from Bacillus anthracis (Zaccai et al., 2008), as the search model. BsFolM was solved with MoRDa (Vagin \& Lebedev, 2015) using BcFolM (PDB entry 5bt9) as the search model. Both structures were refined using iterative cycles of refinement in Phenix (Liebschner et al., 2019) followed by manual structure-rebuilding cycles in Coot (Emsley \& Cowtan, 2004; Emsley et al., 2010). The quality of both structures was checked using MolProbity (Chen et al., 2010). All

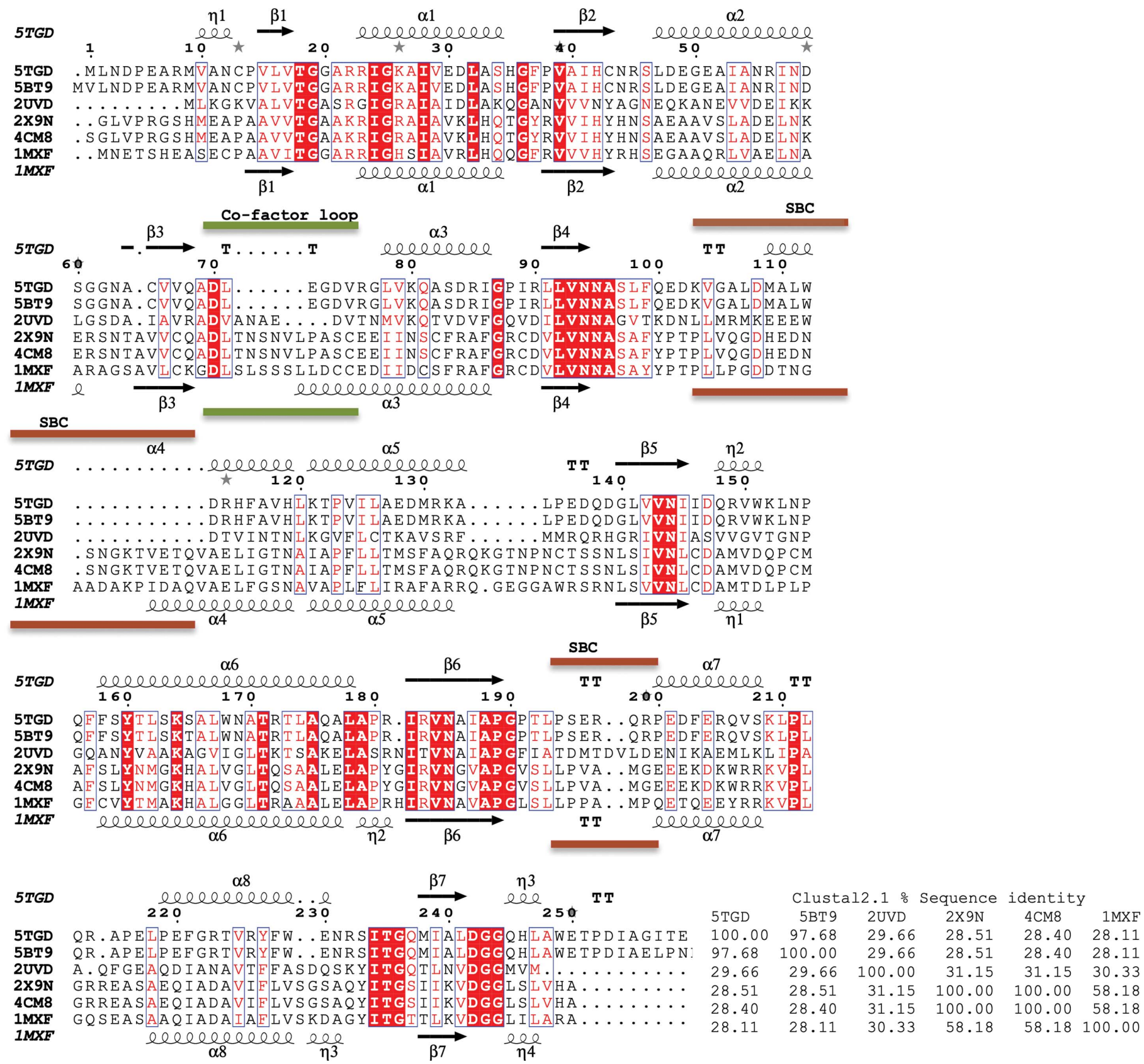

Figure 2

Structural and primary-sequence alignment of FolM alternative dihydrofolate reductase 1 from $B$. suis (PDB entry 5tgd) and B. canis (PDB entry 5bt9) with the molecular-replacement search model 3-oxoacyl-(acyl carrier protein) reductase from Bacillus anthracis (PDB entry 2uvd) and protozoan structures (Trypanosoma brucei pteridine reductase with cyromazine, PDB entry 2x9n; T. brucei pteridine reductase ternary complex with cofactor and inhibitor, PDB entry 4cm8; T. cruzi pteridine reductase, PDB entry 1mxf). The secondary-structure elements are shown as follows: $\alpha$-helices are shown as large coils, $3_{10}$-helices are shown as small coils labeled $\eta, \beta$-strands are shown as arrows labeled $\beta$ and $\beta$-turns are labeled TT. Identical residues are shown on a red background, with conserved residues in red and conserved regions in blue boxes. Regions of greatest variability within the core of the protein are identified with brown lines and labeled SBC due to their proximity to the substrate-binding cavity. 
Table 6

Structure-solution and refinement of FolM alternative dihydrofolate reductase 1 from B. suis (PDB entry 5tgd) and B. canis (PDB entry 5bt9).

\begin{tabular}{lll}
\hline PDB code & $5 \operatorname{tgd}$ & 5 bt9 \\
\hline Resolution range ( $\AA$ ) & $50-1.70(1.74-1.70)$ & $36.15-1.50(1.51-1.50)$ \\
Completeness (\%) & 99.1 & 96.2 \\
$\sigma$ Cutoff & $F>1.34 \sigma(F)$ & $F>1.35 \sigma(F)$ \\
No. of reflections, working set & $116170(8678)$ & $156072(4573)$ \\
No. of reflections, test set & $1785(153)$ & $8084(235)$ \\
Final $R_{\text {cryst }}$ & $0.163(0.2816)$ & $0.169(0.2486)$ \\
Final Prree $_{\text {No. of non-H atoms }}$ & $0.198(0.2825)$ & $0.188(0.2877)$ \\
$\quad$ Protein & 7503 & 7542 \\
$\quad$ Ligand & 214 & 192 \\
$\quad$ Solvent & 748 & 724 \\
$\quad$ Total & 8465 & 8460 \\
R.m.s. deviations & & \\
$\quad$ Bonds ( $\AA$ ) & 0.006 & 0.006 \\
$\quad$ Angles $\left({ }^{\circ}\right)$ & 0.828 & 1.132 \\
Average $B$ factors $\left(\AA^{2}\right)$ & & \\
$\quad$ Protein & 31.9 & 27.7 \\
$\quad$ Ligand & 33.5 & 26.6 \\
$\quad$ Water & 40.0 & 35.1 \\
Ramachandran plot & & \\
$\quad$ Most favored $(\%)$ & 96 & 95 \\
$\quad$ Allowed (\%) & 4 & 5 \\
\hline
\end{tabular}

data-reduction and refinement statistics are shown in Table 6. The $B s$ FolM structure was refined to a resolution of $1.70 \AA$, while that of $B c$ FolM was refined to $1.50 \AA$ resolution. Figures depicting the structure were analyzed and prepared using PyMOL (version 1.5; Schrödinger). Multiple sequence alignments were performed using Clustal Omega (Li, 2003; Sievers et al., 2011). Coordinates and structure factors have been deposited in the Protein Data Bank (https://www.rcsb.org/) as entries 5tgd and $5 \mathrm{bt} 9$ for $B s$ FolM and $B c$ FolM, respectively.

\section{Results and discussion}

The structures of FolM alternative dihydrofolate reductase 1 from B. suis $(B s F o l M)$ and B. canis $(B c$ FolM) were determined in the monoclinic space group $P 2_{1}$ with four monomers in the asymmetric unit (Fig. 1). PDBsum analysis (http:// www.ebi.ac.uk/pdbsum/) indicates that each monomer interacts with three other monomers, with two large interactions and one smaller interaction. The buried surface areas of the interactions are $\sim 1400, \sim 1300$ and $\sim 770 \AA^{2}$ per monomer. These surface areas involve 31,25 and 14 interface amino acids per monomer, respectively. The interface interactions are mostly hydrogen bonds and other nonbonded contacts. The tetramers are similar and superpose with an r.m.s.d. of $\sim 0.5 \AA$ (Fig. 1c). The tetramer is the prototypical short-chain dehydrogenase/reductase (SDR) tetramer, suggesting that the single SEC peak may indeed correspond to a tetramer.

Each monomer has the extended double-Rossmann fold of NADPH-dependent SDRs with a central seven-stranded parallel $\beta$-sheet sandwiched between two pairs of three $\alpha$-helices. Both the $B s$ FolM and $B c$ FolM structures were cocrystallized with a cofactor (NADPH). The monomers are virtually identical, with an r.m.s.d. of $\sim 0.17 \AA$ on superposing all main-chain atoms of both structures (Fig. 1).

The most similar structures to $B s$ FolM and $B c$ FolM were identified by PDBeFold (http://www.ebi.ac.uk/msd-srv/ssm)
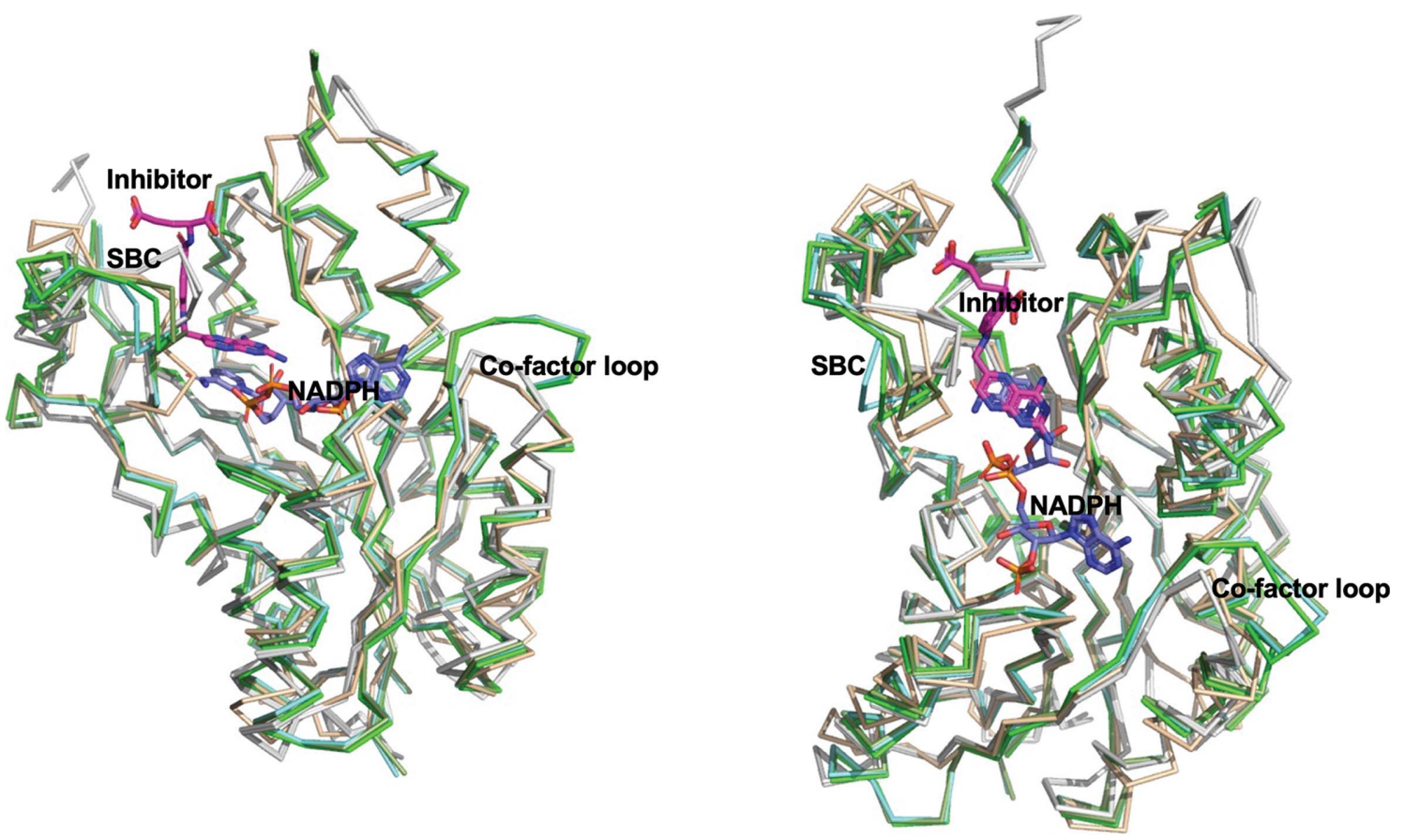

Figure 3

Two views comparing $B s$ FolM and $B c$ FolM monomers with similar structures. The BsFolM and $B c$ FolM monomers (gray) have the prototypical doubleRossmann fold of NADPH-dependent short-chain dehydrogenase/reductases observed in the molecular-replacement search model (tan) and protozoan pteridine reductase (green). The superposed protozoan structures are Trypanosoma brucei pteridine reductase with cyromazine (PDB entry 2x9n; cyan green), T. brucei pteridine reductase ternary complex with cofactor and inhibitor (PDB entry $4 \mathrm{~cm} 8$; dark green) and $T$. cruzi pteridine reductase (PDB entry 1mxf; light green). The cofactor NADPH is shown in blue sticks, while the inhibitor from PDB entry $1 \mathrm{mxf}$ is shown as magenta sticks in the substrate-binding cavity. As in Fig. 2, SBC stands for substrate-binding cavity. 
analysis using the default threshold cutoffs of $70 \%$ for the percentage of the secondary structure of the target chain identified in the query protein and of the secondary structure of the query chain (Krissinel \& Henrick, 2004). The most similar structures are protozoan pteridine reductases (Khalaf et al., 2014; Tulloch et al., 2010; Schormann et al., 2005). These structures share $\sim 29 \%$ sequence identity with BsFolM and $B c$ FolM, and their main-chain $\mathrm{C}^{\alpha}$ atoms align with an r.m.s.d. of $\sim 1.5 \AA$. These protozoan pteridine reductases are more similar to $B s$ FolM and $B c$ FolM than to the structures from Bacillus anthracis (Zaccai et al., 2008), Streptomyces (Wang et al., 2014), Serratia marcescens (Liu et al., 2018), Thermus thermophilus (Asada et al., 2009) or other bacteria.

The $B s$ FolM and $B c$ FolM structures are in the closed conformation with ordered substrate-binding loops, as observed in protozoan pteridine reductases (Khalaf et al.,
2014; Tulloch et al., 2010; Schormann et al., 2005; Schüttelkopf et al., 2005). Despite being identified as the closest structures by PDBeFold, the Trypanosoma proteins share a lower sequence identity to $B s$ FolM and $B c$ FolM than the MR search model from $B$. anthracis (Zaccai et al., 2008), which shares $\sim 30 \%$ sequence identity with both proteins (Fig. 2). Both structures have structural differences from the molecularreplacement search model, the 3-oxoacyl-(acyl carrier protein) reductase (Ba3989) from Bacillus anthracis, and have an r.m.s.d. of $2.12 \AA$ on superposing all main-chain atoms (Fig. 3).

While the cofactor-binding cavities of $B s$ FolM, $B c$ FolM and the Trypanosoma proteins are well conserved, there is a loop insertion (labeled in green; Fig. 2). This loop (labeled the cofactor loop in Fig. 3) points away from the cofactor (NADPH) and aligns well in both $B s$ FolM and $B c$ FolM. Interestingly, this loop is conserved in the protozoan enzymes

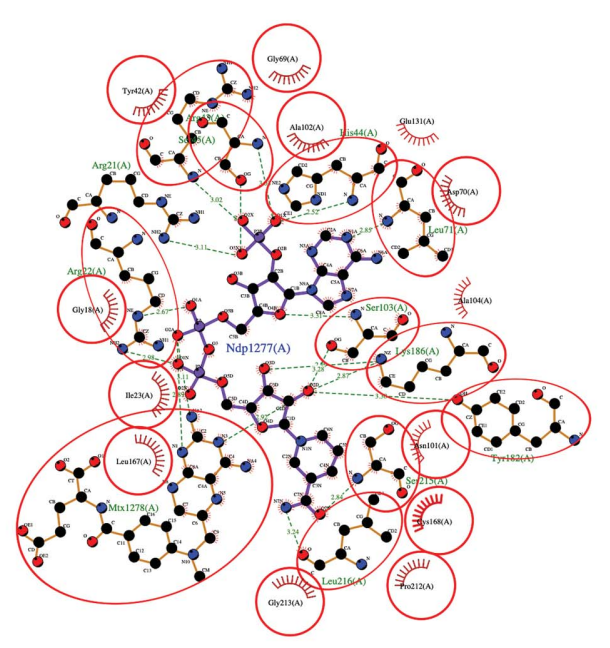

$1 \mathrm{mxf}$

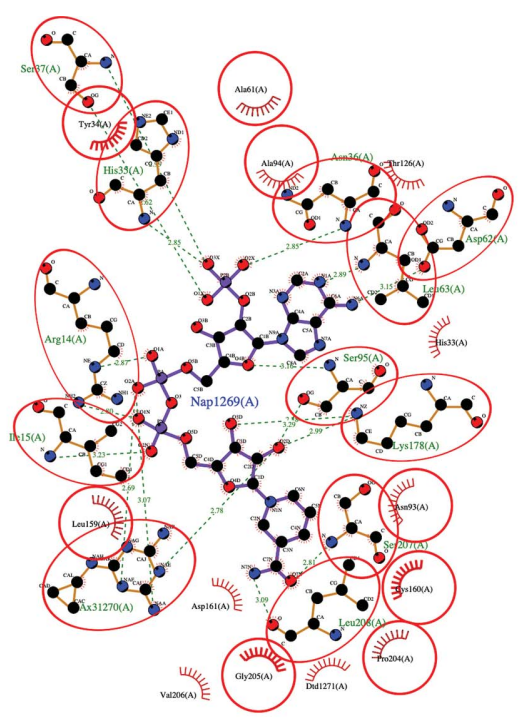

$2 \mathrm{x} 9 \mathrm{n}$

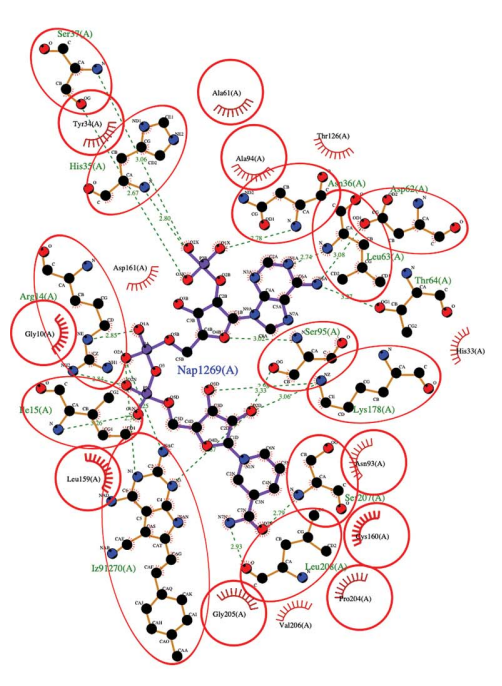

$4 \mathrm{~cm} 8$

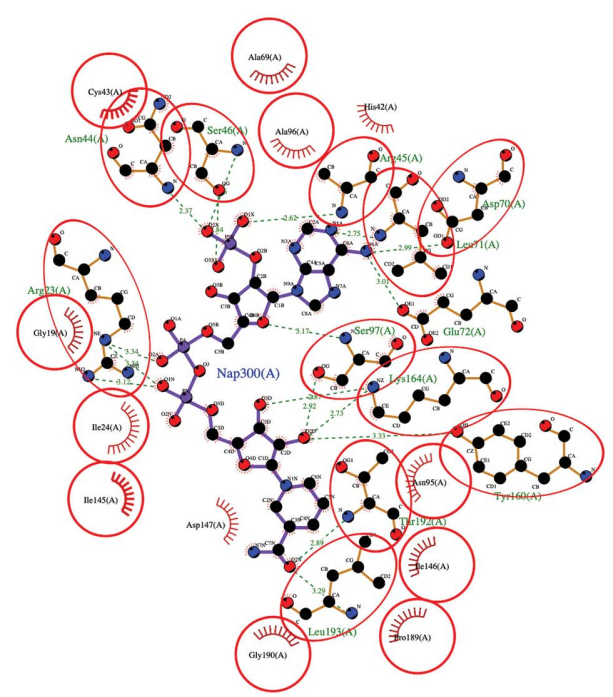

5 bt9

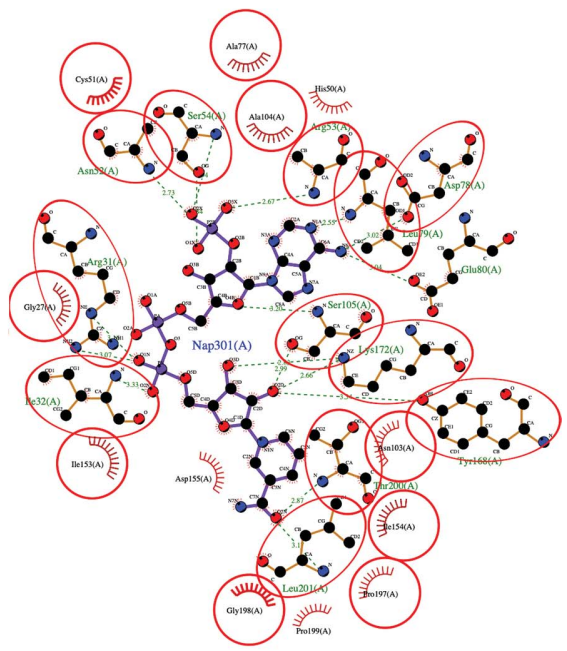

$5 \operatorname{tgd}$

Figure 4

LIGPLOT diagrams reveal well conserved NADPH-binding cavities in FolM alternative dihydrofolate reductase 1 from $B$. suis (PDB entry 5tgd) and B. canis (PDB entry 5bt9), Trypanosoma brucei pteridine reductase with cyromazine (PDB entry 2x9n) T. brucei pteridine reductase in a ternary complex with cofactor and inhibitor (PDB entry $4 \mathrm{~cm} 8$ ) and $T$. cruzi pteridine reductase (PDB entry $1 \mathrm{mxf}$ ). Identical amino-acid residues are circled. 
and forms a $6.5 \AA$ larger cavity than that observed in the Brucella enzymes (both BsFolM and BcFolM; Fig. 3). Apart from this loop region, the cofactor-binding cavity is very similar in these enzymes. Furthermore, the residues involved in NADPH binding are well conserved (Fig. 4).

As expected, the substrate-binding cavity of each protein shows the greatest structural difference (Figs. 2 and 3). This structural variability is believed to allow substrate specificity among SDRs. While the substrates of $B s$ FolM and $B c$ FolM are unknown, their substrate-binding cavities are large enough to accommodate the inhibitors identified by rational therapeutics discovery for human African trypanosomiasis and Chagas disease. There are $>150$ structures of complexes of protozoan pteridine reductases with unique inhibitors deposited in the Protein Data Bank (Khalaf et al., 2014; Tulloch et al., 2010; Schormann et al., 2005) that can serve as starting points for the discovery of therapeutics for brucellosis.

\section{Conclusions}

The high-resolution structures of FolM alternative dihydrofolate reductase 1 from $B$. suis and B. canis have prototypical NADPH-dependent short-chain reductase topology and structural similarity to the well characterized protozoan pteridine reductases. Despite their low sequence identity, their structural similarity to the protozoan pteridine reductases may accelerate drug-repurposing efforts.

\section{Acknowledgements}

The SSGCID consortium is directed by Dr Peter Myler (principal investigator) and comprises many different scientists working at multiple centers towards determining the three-dimensional structures of proteins from biodefense organisms and emerging infectious diseases. In particular, we would like to thank the SSGCID cloning, protein production and X-ray crystallography groups at the Center for Global Infectious Disease Research, the University of Washington and UCB.

\section{Funding information}

This work was supported by federal funds from the National Institute of Allergy and Infectious Diseases (NIAID), National Institutes of Health (NIH), Department of Health and Human Services under Contract No. HHSN272201700059C from 1 September 2017. (SSGCID was funded under NIAID Contract Nos. HHSN272201200025C from 1 September 2012 to 31 August 2017 and HHSN272200700057C from 28 September 2007 to 27 September 2012.) Hampton University students were part of the inaugural Hampton University Chemistry Education and Mentorship Course-based Undergraduate Research (HUChEM CURES) funded by the NIGMS (1U01GM138433).

\section{References}

Ariza, J., Bosilkovski, M., Cascio, A., Colmenero, J. D., Corbel, M. J., Falagas, M. E., Memish, Z. A., Roushan, M. R., Rubinstein, E.,
Sipsas, N. V., Solera, J., Young, E. J., Pappas, G., International Society of Chemotherapy \& Institute of Continuing Medical Education of Ioannina (2007). PLoS Med. 4, e317.

Asada, Y., Endo, S., Inoue, Y., Mamiya, H., Hara, A., Kunishima, N. \& Matsunaga, T. (2009). Chem. Biol. Interact. 178, 117-126.

Aslanidis, C. \& de Jong, P. J. (1990). Nucleic Acids Res. 18, 60696074.

Blaise, M., Van Wyk, N., Banères-Roquet, F., Guérardel, Y. \& Kremer, L. (2017). Biochem. J. 474, 907-921.

Bryan, C. M., Bhandari, J., Napuli, A. J., Leibly, D. J., Choi, R., Kelley, A., Van Voorhis, W. C., Edwards, T. E. \& Stewart, L. J. (2011). Acta Cryst. F67, 1010-1014.

Chen, V. B., Arendall, W. B., Headd, J. J., Keedy, D. A., Immormino, R. M., Kapral, G. J., Murray, L. W., Richardson, J. S. \& Richardson, D. C. (2010). Acta Cryst. D66, 12-21.

Choi, R., Kelley, A., Leibly, D., Nakazawa Hewitt, S., Napuli, A. \& Van Voorhis, W. (2011). Acta Cryst. F67, 998-1005.

Ducrotoy, M., Bertu, W. J., Matope, G., Cadmus, S., Conde-Álvarez, R., Gusi, A. M., Welburn, S., Ocholi, R., Blasco, J. M. \& Moriyón, I. (2017). Acta Trop. 165, 179-193.

Ducrotoy, M. J., Conde-Álvarez, R., Blasco, J. M. \& Moriyón, I. (2016). Vet. Immunol. Immunopathol. 171, 81-102.

Emsley, P. \& Cowtan, K. (2004). Acta Cryst. D60, 2126-2132.

Emsley, P., Lohkamp, B., Scott, W. G. \& Cowtan, K. (2010). Acta Cryst. D66, 486-501.

Figueiredo, P. de, Ficht, T. A., Rice-Ficht, A., Rossetti, C. A. \& Adams, L. G. (2015). Am. J. Pathol. 185, 1505-1517.

Godfroid, J., Al Dahouk, S., Pappas, G., Roth, F., Matope, G., Muma, J., Marcotty, T., Pfeiffer, D. \& Skjerve, E. (2013). Comp. Immunol. Microbiol. Infect. Dis. 36, 241-248.

Godfroid, J., Garin-Bastuji, B., Saegerman, C. \& Blasco, J. M. (2013). Rev. Sci. Tech. OIE, 32, 27-42.

Godfroid, J., Scholz, H. C., Barbier, T., Nicolas, C., Wattiau, P., Fretin, D., Whatmore, A. M., Cloeckaert, A., Blasco, J. M., Moriyon, I., Saegerman, C., Muma, J. B., Al Dahouk, S., Neubauer, H. \& Letesson, J. J. (2011). Prev. Vet. Med. 102, 118-131.

Kabsch, W. (2010). Acta Cryst. D66, 125-132.

Khalaf, A. I., Huggan, J. K., Suckling, C. J., Gibson, C. L., Stewart, K., Giordani, F., Barrett, M. P., Wong, P. E., Barrack, K. L. \& Hunter, W. N. (2014). J. Med. Chem. 57, 6479-6494.

Krissinel, E. \& Henrick, K. (2004). Acta Cryst. D60, 2256-2268.

Levin, I., Giladi, M., Altman-Price, N., Ortenberg, R. \& Mevarech, M. (2004). Mol. Microbiol. 54, 1307-1318.

Li, K. B. (2003). Bioinformatics, 19, 1585-1586.

Liebschner, D., Afonine, P. V., Baker, M. L., Bunkóczi, G., Chen, V. B., Croll, T. I., Hintze, B., Hung, L.-W., Jain, S., McCoy, A. J., Moriarty, N. W., Oeffner, R. D., Poon, B. K., Prisant, M. G., Read, R. J., Richardson, J. S., Richardson, D. C., Sammito, M. D., Sobolev, O. V., Stockwell, D. H., Terwilliger, T. C., Urzhumtsev, A. G., Videau, L. L., Williams, C. J. \& Adams, P. D. (2019). Acta Cryst. D75, 861-877.

Liu, J.-S., Kuan, Y.-C., Tsou, Y., Lin, T.-Y., Hsu, W.-H., Yang, M.-T., Lin, J.-Y. \& Wang, W.-C. (2018). Sci. Rep. 8, 2316.

Long, F., Vagin, A. A., Young, P. \& Murshudov, G. N. (2008). Acta Cryst. D64, 125-132.

Megersa, B., Biffa, D., Abunna, F., Regassa, A., Godfroid, J. \& Skjerve, E. (2011). Trop. Anim. Health Prod. 43, 651-656.

Moreno, E. (2014). Front. Microbiol. 5, 213.

Myler, P. J., Stacy, R., Stewart, L., Staker, B. L., Van Voorhis, W. C., Varani, G. \& Buchko, G. W. (2009). Infect. Disord. Drug Targets, 9, 493-506.

Pampa, K. J., Lokanath, N. K., Kunishima, N. \& Rai, R. V. (2014). Acta Cryst. D70, 994-1004.

Schormann, N., Pal, B., Senkovich, O., Carson, M., Howard, A., Smith, C., DeLucas, L. \& Chattopadhyay, D. (2005). J. Struct. Biol. 152, 64-75.

Schüttelkopf, A. W., Hardy, L. W., Beverley, S. M. \& Hunter, W. N. (2005). J. Mol. Biol. 352, 105-116. 
Serbzhinskiy, D. A., Clifton, M. C., Sankaran, B., Staker, B. L., Edwards, T. E. \& Myler, P. J. (2015). Acta Cryst. F71, 594-599.

Sievers, F., Wilm, A., Dineen, D., Gibson, T. J., Karplus, K., Li, W., Lopez, R., McWilliam, H., Remmert, M., Söding, J., Thompson, J. D. \& Higgins, D. G. (2011). Mol. Syst. Biol. 7, 539.

Stacy, R., Begley, D. W., Phan, I., Staker, B. L., Van Voorhis, W. C., Varani, G., Buchko, G. W., Stewart, L. J. \& Myler, P. J. (2011). Acta Cryst. F67, 979-984.

Studier, F. W. (2005). Protein Expr. Purif. 41, 207-234.
Tulloch, L. B., Martini, V. P., Iulek, J., Huggan, J. K., Lee, J. H., Gibson, C. L., Smith, T. K., Suckling, C. J. \& Hunter, W. N. (2010). J. Med. Chem. 53, 221-229.

Vagin, A. \& Lebedev, A. (2015). Acta Cryst. A71, s19.

Wang, H., Zhang, H., Zou, Y., Mi, Y., Lin, S., Xie, Z., Yan, Y. \& Zhang, H. (2014). PLoS One, 9, e97996.

Zaccai, N. R., Carter, L. G., Berrow, N. S., Sainsbury, S., Nettleship, J. E., Walter, T. S., Harlos, K., Owens, R. J., Wilson, K. S., Stuart, D. I. \& Esnouf, R. M. (2008). Proteins, 70, 562-567. 